\title{
Continuous Non-Invasive Sodium Monitoring in Extracorporeal Circuits
}

\author{
Marc Berger, Daniel Niebuhr, Maria Allers, Stefan Zimmermann \\ Leibniz Universität Hannover, Institute of Electrical Engineering and Measurement Technology, \\ Department of Sensors and Measurement Technology, Appelstr. 9A, 30167 Hannover, Germany \\ berger@geml.uni-hannover.de
}

\begin{abstract}
:
Dialysis is a blood purification therapy indicated by kidney failure, where blood is pumped via an extracorporeal circuit into a dialyzer consisting of a semi-permeable membrane separating blood and the dialysate. Due to the concentration gradients across this membrane, toxins can be removed from the blood by diffusion and the electrolyte balance can be regulated [1]. However, excessive changes of osmotic substances such as electrolytes can be critical. For instance, a rapid or excessive loss of sodium in blood, and thus a drop in plasma osmolarity, can cause overhydrating of cells, cardiovascular instability and disequilibrium syndrome with headache, muscle cramps and fatigue symptoms. On the other hand, an inefficient loss of sodium can cause increased thirst, hypertension and risk of pulmonary edema [2-5]. Especially for critically ill patients with acute kidney injury, any additional stress during dialysis has to be avoided. A dialysate with individually adjusted electrolytic concentration can prevent such complications. Thus, it is necessary to monitor the plasma electrolyte concentrations and other osmotic substances during dialysis treatment [6]. However, the required hemocompatibility is often difficult with invasive in-line measurement methods [7]. In this work, we present a new approach to non-invasively monitor sodium based on a differential transformer. The output voltage of this transformer depends on the conductivity of the medium [8]. First measurements show a linear correlation between sodium concentration and output voltage.
\end{abstract}

Key words: continuous sodium monitoring, non-invasive in-line monitoring, differential transformer, individualized hemodialysis, extracorporeal circuits

\section{Introduction and Experimental Setup}

A differential transformer usually consists of three coils on a ferrite core. We use a ferrite core with a relative permeability of $\mu_{\mathrm{r}}=300$. Additionally, we add a fourth compensation coil $L_{c}$ to the differential transformer, which eliminates asymmetries in the setup by active compensation. Fig. 1 shows the experimental setup. The primary coil $L_{p}$, excited with an alternating voltage $U_{p}$, generates a primary flux through the secondary coils $L_{s 1}$ and $L_{s 2}$ as well as the medium. Due to the opposite direction of winding and the same inductivity of the two secondary coils, no output voltage $U_{s}$ should be induced by the primary flux. However, small asymmetries in the setup lead to an induced voltage, which can be actively reduced by the additional compensation coil $L_{c}$. Inside the medium, the primary flux generates displacement and eddy currents causing a secondary flux. Since the medium is closer to the secondary coil $L_{s 1}$ compared to $L_{s 2}$, a higher voltage is induced into $L_{s 1}$ resulting in an output voltage $U_{s}$. The voltage $U_{s}$ can be calculated according to Eq. (1):
$U_{s}=K_{1}\left(\omega^{2}\right) \cdot \epsilon_{r}^{\prime}-j \cdot K_{2}(\omega) \cdot\left(\omega \cdot \epsilon_{r}^{\prime \prime}+\kappa\right)$

where $j$ is the imaginary unit, $\omega$ is the angular frequency, $\mathrm{K}_{1}$ and $\mathrm{K}_{2}$ are frequency-dependent constants, $\varepsilon$ 'is the polarizability of the medium, $\varepsilon$ " describes the dielectric losses and $K$ is the electrical conductivity [8].

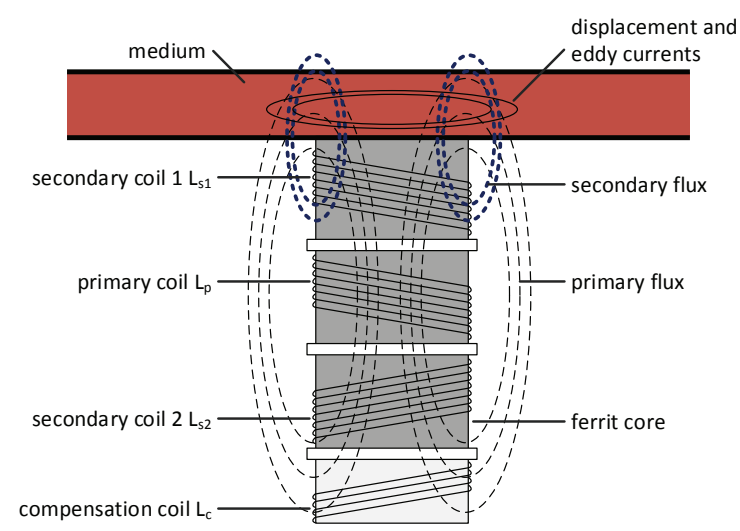

Fig. 1. Schematic setup of the differential transformer. 
Assuming a linear correlation between the conductivity of blood plasma and the sodium concentration, which is a sound assumption due to the significantly higher sodium concentration compared to the other electrolytes, it is possible to determine the sodium concentration from the inductively measured electrical conductivity of blood, when the dielectric losses $\varepsilon$ " are zero $[3,9,10]$.

\section{Results}

In preliminary experiments, the sensor response is investigated for different sodium concentrations in DI-water. In addition, the test solutions contain $5 \mathrm{mmol} / \mathrm{l}$ urea comparable to blood urea concentrations to account for interfering effects. Fig. 2 illustrates the imaginary part of the output voltage $U_{s}$. As predicted by Eq. (1), a linear correlation between the sodium concentration and output voltage $U_{s}$, with a coefficient of determination $R^{2}=0.99$ and a sensitivity $S=2.08 \mathrm{mV} / \mathrm{mol} / \mathrm{l}$, results after an initial step. The initial step only occurs at very low concentrations and may be the result of frequency and concentration dependent dielectric losses [11].

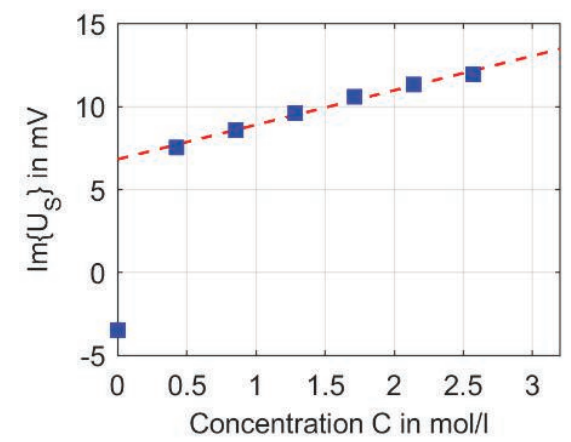

Fig. 2. Imaginary part of the output voltage $U_{s}$ versus sodium concentration in a $5 \mathrm{mmol} / \mathrm{l}$ urea solution.

In order to exclude possible cross-sensitivities, the influence of other substances on the imaginary part of $U_{s}$, which can significantly change during dialysis treatment, was investigated. For example, Fig. 3 shows the influence of urea in a $140 \mathrm{mmol} / \mathrm{l}$ sodium chloride solution with a cross-sensitivity of just $0.07 \mathrm{mV} / \mathrm{mol} / \mathrm{l}$ leading to an absolut error of approximately $\pm 1.35 \mathrm{mmol}$ sodium for possible changes of urea of $40 \mathrm{mmol} / \mathrm{l}$ during dialysis treatment. This gives a relative error of just $1 \%$. Similar results apply for glucose.

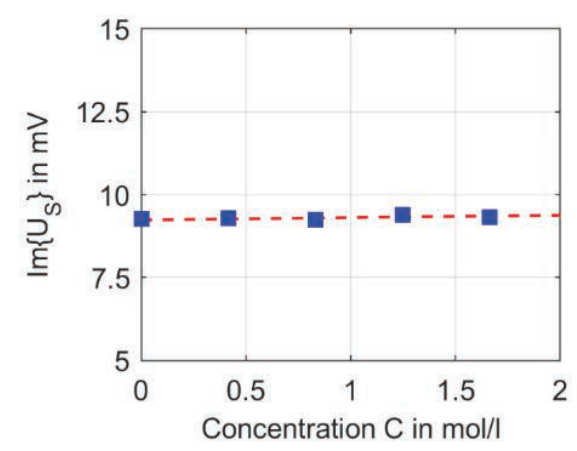

Fig. 3. Imaginary part of the output voltage $U_{s}$ versus urea concentration in a $140 \mathrm{mmol} / \mathrm{l} \mathrm{NaCl}$ solution.
We conclude that the actively compensated differential transformer is a promising approach for noninvasive monitoring of the sodium concentration in extracorporeal circuits, which is especially interesting during hemodialysis.

\section{Acknowledgments}

Sponsored by the German Federal Ministry of Education and Research (BMBF) under the grant 13GW0085B.

\section{References}

[1] M. Sivalingam, K. Farrington, Haemodialysis, Medicine 35, 461-465 (2007); doi: 10.1016/j.mpmed.2007.05.005.

[2] S. Stiller, E. Bonnie-Schorn, A. Grassmann, I. Uhlenbusch-Körwer, H. Mann, A Critical Review of Sodium Profiling for Hemodialysis, Seminars in Dialysis 14, 337-347 (2001); doi: 10.1046/j.1525139X.2001.00086.x.

[3] F. Locatelli, V. La Milia, L. Violo, L. Del Vecchio, S. Di Filippo, Optimizing haemodialysate composition, Clinical kidney journal 8, 580-589 (2015); doi: 10.1093/ckj/sfv057.

[4] B. F. Palmer, Individualizing the Dialysate in the Hemodialysis Patient, Seminars in Dialysis 14, 41-49 (2001); doi: 10.1046/j.1525-139x.2001.00013.x.

[5] F. M. de Paula, A. J. Peixoto, L. V. Pinto, D. Dorigo, P. J. M. Patricio, S. F. F. Santos, Clinical consequences of an individualized dialysate sodium prescription in hemodialysis patients, Kidney international 66, 1232-1238 (2004); doi: 10.1111/j.15231755.2004.00876.x.

[6] M. K. Sharma, F. P. Wieringa, A. J. H. Frijns, J. P. Kooman, On-line monitoring of electrolytes in hemodialysis: on the road towards individualizing treatment, Expert review of medical devices 13, 933-943 (2016); doi: 10.1080/17434440.2016.1230494.

[7] V. G. Gavalas, M. J. Berrocal, L. G. Bachas, Enhancing the blood compatibility of ion-selective electrodes, Analytical and bioanalytical chemistry 384, 65-72 (2006); doi: 10.1007/s00216-005-0039-0.

[8] T. Reinecke, P. Biechele, M. Sobocinski, H. Suhr, K. Bakes, D. Solle, H. Jantunen, T. Scheper, S. Zimmermann, Continuous noninvasive monitoring of cell growth in disposable bioreactors, Sensors and Actuators B: Chemical 251, 1009-1017 (2017); doi: 10.1016/j.snb.2017.05.111.

[9] F. Locatelli, S. Di Filippo, C. Manzoni, Relevance of the conductivity kinetic model in the control of sodium pool, Kidney international 58, S89-S95 (2000); doi: 10.1046/j.1523-1755.2000.07611.x.

[10] A. Tura, S. Sbrignadello, E. Mambelli, P. Ravazzani, A. Santoro, G. Pacini, Sodium concentration measurement during hemodialysis through ion-exchange resin and conductivity measure approach: In vitro experiments, PloS one 8, e69227 (2013); doi: 10.1371/journal.pone.0069227.

[11] M. Buehler, D. Cobos, K. Dunne, eds., Dielectric Constant and Osmotic Potential from Ion-Dipole Polarization Measurements of $\mathrm{KCl}$ - and $\mathrm{NaCl}$-doped Aqueous Solutions.; June 2011. 